\title{
Technical Research on High Performance Fully Compatible FMC Fast Connector
}

\author{
Yinghui Liu, Qiufei Yao, Yuanyuan Wu, Jianhua Sun, Lihua Li, Yi Xu \\ Zhongtian Broadband Technology CO., LTD, Nantong, China \\ Email address: \\ liuyinghui@chinaztt.com (Yinghui Liu),yaoqf@chinaztt.com (Qiufei Yao),wuyuanyuan@ chinaztt.com (Yuanyuan Wu), \\ sunjhua@chinaztt.com (Jianhua Sun), lilh@chinaztt.com (Lihua, Li), gary.xu@zttcable.com (Yi Xu)
}

\section{To cite this article:}

Yinghui Liu, Qiufei Yao, Yuanyuan Wu, Jianhua Sun, Lihua Li, Yi Xu. Technical Research on High Performance Fully Compatible FMC Fast Connector. International Journal of Information and Communication Sciences. Vol. 5, No. 4, 2020, pp. 41-45.

doi: $10.11648 /$ j.ijics.20200504.11

Received: November 17, 2020; Accepted: December 21, 2020; Published: December 25, 2020

\begin{abstract}
With the rapid development of the optical communication industry, the implementation of national policies, the increase of broadband access fiber-to-the-home investment, and the continuously increased requirements for the important components in the construction and deployment of FTTH system solutions of the communication industry, fiber connection has become an important link in FTTH. The fast connector has become the main method of optical connection, and the emergence of the fast connector solves the problem that the optical fiber can only be spliced. This article mainly introduces the basic characteristics and technical parameters of high-performance fully compatible FMC fast connectors which is an important device in the construction and deployment of the current FTTH system solution. By implementing the basic characteristic of "high performance" and "fully compatible", it can meet all the terminal connection demand under various FTTH constructions deployment. And at the same time, this article briefly explains the convenience of construction and practical application of the FMC fast connectors. Through the optimization and improvement of various performances, the connector product has become an important technology for future FTTH network construction. It is suitable for terminal connection in various FTTH construction deployment environments, reducing on-site construction costs, improving assembly efficiency, providing convenience and services, and meeting the needs of customer terminal connection with different usage habits.
\end{abstract}

Keywords: High-performance, Fully Compatibel, Fast Connectors, Convenience of Construction, Practical Application

\section{Introduction}

With the rapid development of the optical communication industry, the implementation of national policies, the increase of broadband access fiber-to-the-home investment, and the continuously increased requirements for the important components in the construction and deployment of FTTH system solutions of the communication industry, fiber connection has become an important link in FTTH, And the fast connector has become the main method of optical connection. Field-Mountable Optical Fiber Connector (Hereinafter referred to as FMC) is an optical fiber movable connector that can be directly assembled on the sheath of the optical fiber or cable at the construction site. FMC fast connector is a product that can move the manufacturing process of the connector to the construction site, use the mechanical connection technology to assemble directly and can be completed quickly. [1]

The pre-installed field connector is a field connector in which a section of optical fiber is pre-embedded by the factory and the end face of optical fiber is polished. It is a connector with multiple connection points. The fiber connection point is in the V-groove, and the matching gel is injected in the groove. The matching gel can make up some small defects of the cutting end face and make it with low loss. The pre-embedded optical fiber after grounding can better connect with the on-site cutting optical fiber to achieve the expected light transmission effect. The key technology of the pre-installed connector is the high-precision V-groove and the matching gel. $[2,3]$

Based the concept of "Connect the World with Optical Fiber, Build a Better Future", Jiangsu Zhongtian Technology CO., LTD (Hereinafter referred to as "ZTT Group") encourage and inspire technical craftsmen to develop and create products that 
are in line with international standard and create better \&new life. That is, to meet the high-end international users' excellent performance and structurally compatible use needs. A survey of multiple domestic and overseas supply and potential markets revealed that the usage habits of users differs and it also differs among the different areas. International high-end customers have higher demand for pre-installed field optical connectors. Therefore, the development of optical connectors with the characteristic of easy installation, stable performance, and compatible structure has become an important link in improving and optimizing the construction solution of fiber to the home. [4] At present, the quality of connectors supplied by different manufacturers at home and abroad is uneven, and the qualification rate is relatively low. There exists many problems in the on-site constructions, such as V-groove switch locked fiber unable to enter, broken tail, low assembly yield rate and high optical fiber loss after being connected and so on. Through the analysis and research of many times, with the ways of improving the connector design, studying and choosing the new raw materials, improving the inspection methods to ensure the accuracy of testing result and optimizing the manufacturing process to improve product indicators and precision, ZTT group has developed and produced a new type of FMC connectors of excellent indicators, stable performance, sophisticated technology, high yield rate and full compatibility.
This article mainly introduces a high-performance and fully compatible FMC fast connector, which is also an important device in the construction and deployment of the current FTTH system solution. By implementing the basic characteristic of "high performance" and "fully compatible", it can meet all the terminal connection demand under various FTTH constructions deployment. On the other hand, ZTT group is customer-centric and actively responding to the changing trend of customer demand. Through the new products development, ZTT Group provides customers with convenience and services to control construction costs, improve assembly efficiency, and satisfy customers' needs. In addition, this article also briefly describes its technical parameters, construction convenience and practical application.

\section{Overview of High-performance fully Compatible FMC Fast Connectors}

\subsection{Structure of High-performance and Fully Compatible FMC Fast Connector}

The structure is shown in the "Figure 1": The key components of this fast connector are V-groove, pre-installed fiber and ferrule, fiber clamp, locking latch. And the key material is matching gel.

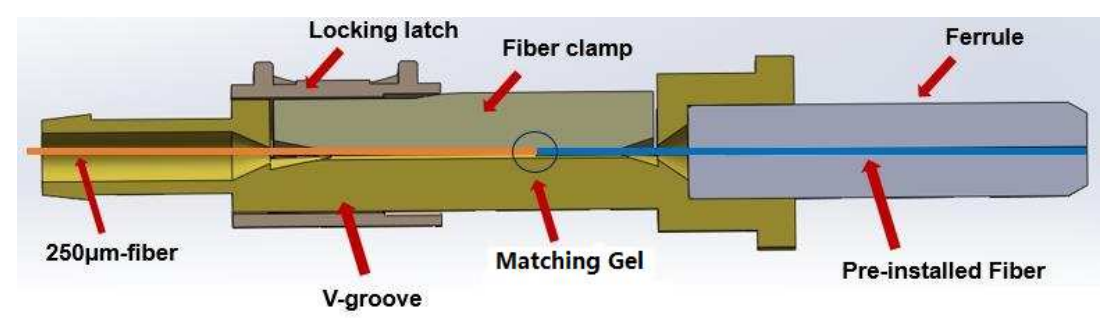

Figure 1. There are seven key components here.

\subsection{The Principle of High-performance and Fully Compatible FMC Fast Connector}

The principle is shown in the figure below. When lock buckle slides to the "On" state, the construction splicing fiber can be reliably aligned with the embedded fiber through the clever design of the product structure. When the lock buckle slides to the "OFF" state, it realizes the locking of aligned optical fiber and ensure the successful of optical fiber connection.

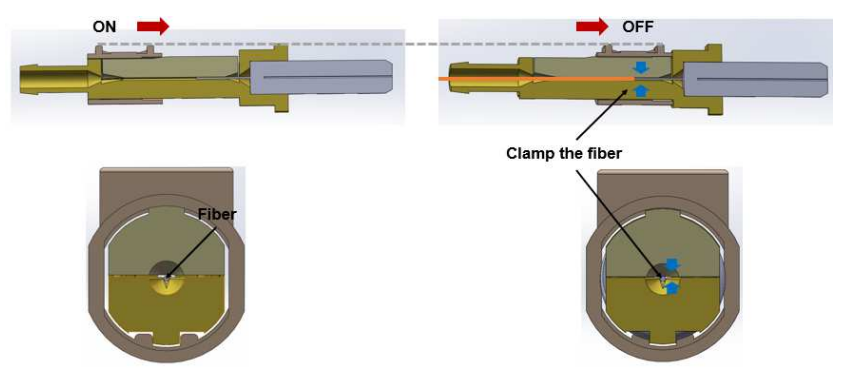

Figure 2. It shows how to align and lock the optical fiber.

\subsection{Technical Parameters of High-performance and Fully Compatible FMC Fast Connector}

The typical features of FMC fast Connector s introduced in this article are as follows:

(1) Working temperature: $-40^{\circ} \mathrm{C}-85^{\circ} \mathrm{C}$

(2) Working temperature and humidity: $95 \%\left(30^{\circ} \mathrm{C}\right)$;

(3) Structure compatible: $2.0 \mathrm{~mm}$ Round optical cable, $3.0 \mathrm{~mm}$ Round optical cable, $2.0 * 3.0 \mathrm{~mm}$ Indoor and outdoor drop cable, $2.0 * 1.6 \mathrm{~mm}$ Indoor and outdoor drop cable; [5]

(4) Mechanical durability: 500 times;

(5) Geometrical requirement of optical fiber:

Fiber height: $-100 \sim+50 \mathrm{~nm}$;

Radius of curvature: $10 \sim 25 \mathrm{~mm}$;

Vertex offset: 0 50um;

APC Angle: $8.0 \pm 0.2^{\circ}$; [6]

(6) Low temperature life (online test):

Temperature: $-40^{\circ} \mathrm{C}$; Continued: 96 hours;

(7) Thermal life (online test):

Temperature: $85^{\circ} \mathrm{C}$;

Continued: 96 hours; [7] 
(8) High and low temperature cycle (online test):

Temperature: $-40^{\circ} \mathrm{C}-85^{\circ} \mathrm{C}$; Continued: 168 hours;

Temperature speed: $1{ }^{\circ} \mathrm{C} /$ minute;

Cycles: 21 times; [8]

(9) Wet aging (online test)

Temperature: $75^{\circ} \mathrm{C}$

Humidity: $95 \%$

Continued: 96 Hours;

(10) Water immersion test (Online test):

Room temperature

Continued: 168 Hours;

(11) Optical performance requirements insertion and return loss:

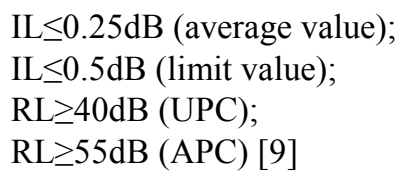

(12) Environmental performance:

Use environmentally friendly materials to meet RoHS requirements and prevent pollution

\section{Technical Research on High-performance and Fully Compatible FMC Fast Connector}

\subsection{Technical Requirements for High-performance and Fully Compatible FMC Fast Connector}

Some proportions of telecom operators only require that the change of insertion return loss before and after the test meet the standard for environmental performance. While high-end customers require real-time online for the environmental performance of fast-connect products to monitor the change of optical performance during the test, and the change is required to be controlled within $\leq 0.3 \mathrm{~dB}$. [10]

It is extremely important to identify the key technical difficulties. At the same time, it is necessary to consider from multiple dimension to ensure the rationality and accuracy of the program design. Different from traditional fast connectors, in addition to matching indoor and outdoor drop cable, it also needs to be compatible with $2.0 / 3.0 \mathrm{~mm}$ round optical cables. [11]In addition, in terms of environmental performance, there are still many technical difficulties in how to achieve the online variation. [12] How to realize the development of a fully compatible optical cable fast connector through structural design, and ensure that the mechanical performance meets the requirements?

\subsection{Detection Method of High-performance and Fully Compatible FMC Fast Connector}

The traditional online monitoring system for environmental performance based on manual construction has disadvantages such as limited channels, manual waste, and data errors. Therefore, the online monitoring system needs to be reselected and optimized. Considering the market acceptance, testing applicability, technical expertise, economic rationality, after-sales service, etc., using an advanced online environmental performance monitoring system, the entire test can achieve 16 channels at one time without manual plugging, testing optical performance and recording the test value. The system can record and save the dynamic changes of the data in real time, the database is enlarged, and then through the analysis of the collected data, the fluctuation of the data is relatively stable. [13]

\subsection{Technical Research on High-performance and Fully Compatible FMC Fast Connector}

\subsubsection{Realize the Automation of Injecting Matching gel and} Improve the Uneven Filling of Matching Gel

Based on the sample size of 200pcs FMC fast connector semi-finished products and different workers' operation proficiency level, we verify the performance indicators. Through the data analysis in the above figure, after realizing the automatic matching gel injection, employees of different proficiency levels have little effect on the performance indicators (Insertion Loss), which solves the bottle neck of human factors control and improves the matching gel filling uneven problems.

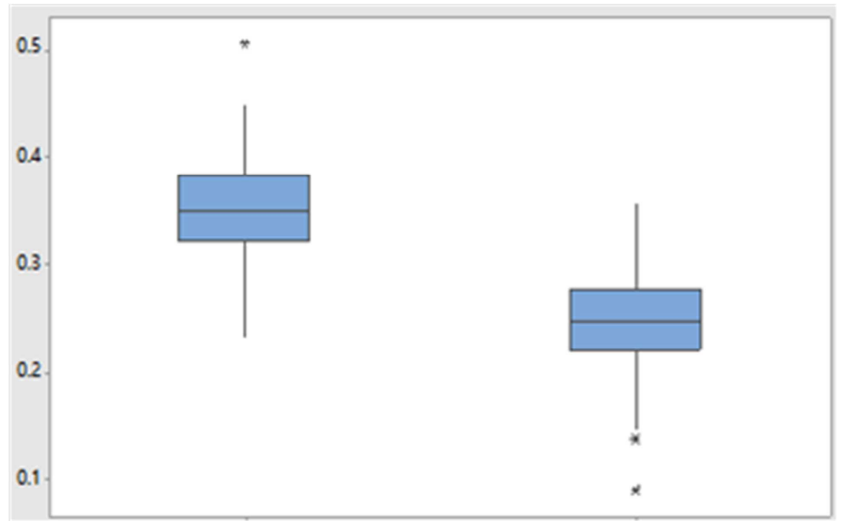

Figure 3. The matching gel control between 0.1 0.5.

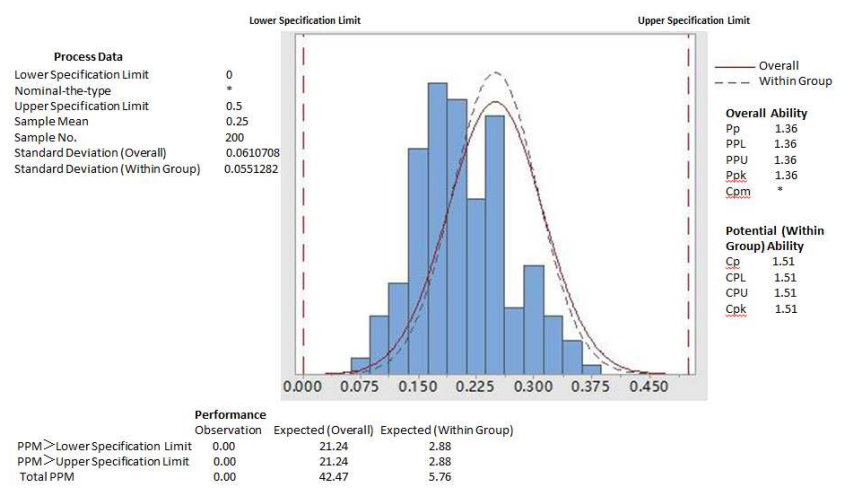

Figure 4. The improved process make the product quality much more stable.

\subsubsection{Characteristic Analysis of Matching Gel}

From the comprehensive consideration of oil-water separation rate, refractive index, life evaluation and other aspects, we change the key raw materials to a new brand. At the same time, two senior technical engineers from the new 
brand supplier have the statement that under normal condition of use, refractive index matching materials can guarantee 25 years of stability and reliability. And they also have the paper about the matching gel life assessment concerned by customers.

\subsubsection{Embedded Fiber End Face Dirty Cracks, Embedded Fiber Deep Processing, Arc Treatment for Embedded Fiber Cutting End Face}

Through the equipment transformation, the fusion splicer was transformed to arc processing equipment. In the actual operation process, considering the imaging clarity and current parameter setting accuracy, etc., we jointly developed the arc processing equipment version 2.0 with equipment manufacturer. [14] With the debugging rod, the new equipment can clearly image the cutting end face of the embedded fiber. The current size adjustment is set with 5 levels. Finally by the statistical analysis of the test data, the embedded fiber cutting end process parameters formed by arc processing, when the current controlled within 3-4 levels, have the industry-leading level of pre-embedded fiber end face treatment.

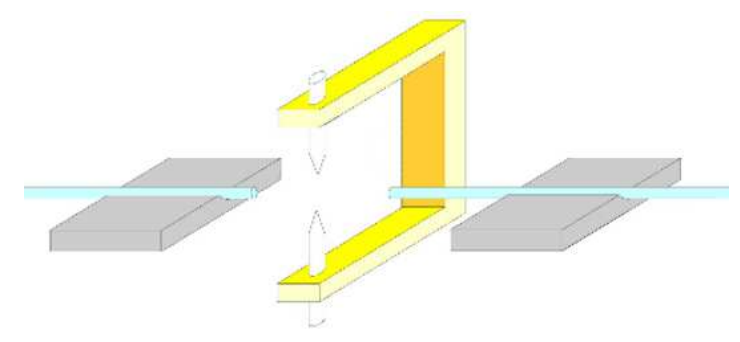

Figure 5. Using the Principle of Electrode Discharge to Breakdown Arc.

\subsubsection{Material Switching of Fast Connector}

In response to the problem of optical variation excessive for environmental online monitoring, project team conducted a grouped test study on FMC fast connectors' materials, under the premise of confirming the clamping structure of the product tail.

The connectors of three different raw materials were assembled and the environmental performance was monitored online. According to the test result, the PEI material has outstanding performance advantages. During the high and low temperature cycle, it has little impact for V-groove fiber locking and clamping of the optical cable tail. During the online monitoring process of the entire temperature cycle test, the change of insertion and return loss is within the technical requirements. [15]

\begin{tabular}{|c|c|c|}
\hline PC & PP+GF & PEI \\
\hline & & \\
\hline & & \\
\hline NG in The Experiment & NG in The Experiment & PASS \\
\hline
\end{tabular}

Figure 6. The experiment result for three different materials.

\section{Conclusion}

High-performance and Fully Compatible FMC Fast Connector is an important technology for future FTTH network construction. The product can be compatible with 2.3 , 3. $\mathrm{mm}$ round optical cables and $2.0 * 3.0 \mathrm{~mm}, 2.0 * 1.6 \mathrm{~mm}$ indoor and outdoor drop cables during the construction process. To a certain extent, it breaks through the limitations of the traditional FMC fast connectors product structure, satisfies the connection of optical cables terminals in various FTTH construction deployment environments, and solves the difficulties caused by splicing. In addition, the product matches four optical cables with different structures and passes the online environmental performance test. It also meets the standard of insertion and return loss.

Looking forward to the future, we should focus on the core competiveness of new products, design and product more stable, reliable and convenient products to improve the quality of human life and make more contributions to the sustainable development of mankind.

\section{References}

[1] Xiaoyan Jian, Shaoxian Chen, Yu Zhang, et al. Quality analysis of prefabricated field assembled optical fiber connectors $[\mathrm{J}]$. Guangdong Communication Technology, 2013 (07): 69-72.

[2] Yaguo Xu, Structure Design of Mechanical Field Optical Fiber Fast Device. Value Engineering, 1006-4311(2014)34-0033-03.

[3] Jian Liu, Shaoxian Chen, Renmei Mo, Yuantao Zhou, Research on Relevant Technical Standards for Mechanical Field Fast Connectors and Cold Fusion Connectors. Guangdong Communication Technology, July, 2014.

[4] Ruolin Zhang, Investigation of preset and Non-preset Fiber Optic Connector. Thesis Submitted to Nanjing University of Post and Telecommunications for the Degree of Master of Engineering, February 2013.

[5] YD/T 2341.1-2011 Field-mountable optical fiber connectors part 1: mechanical type, Released on December 20, 2011.

[6] Q/CT 2299-2010, Technical Requirements for Field-mountable Optical Connector of China Telecom, Released on December 2010.

[7] YD/T 1272.1-2018 Optical Fiber Connector Part I Type LC, The Ministry of Information Industry of the People's Republic of China, 2018.

[8] YD/T 1272.3-2015 Optical Fiber Connector Part III Type S, The Ministry of Information Industry of the People's Republic of China, 2015.

[9] YD/T 1272.3-2015 Optical Fiber Connector Part IV Type FC, The Ministry of Information Industry of the People's Republic of China, 2015.

[10] Jingzhong Shen, Baoyan Li The selection and precautions of FTTH field assembled optical fiber connector [J]. Intelligent Building and Urban Information, 2013 (10): 18-21. 
[11] Daqing Zeng, Yi Zhao, Quality analysis of end face of single connection point mechanical field assembled optical fiber connector [J]. Telecommunications Technology, 2017 (2).

[12] Jian liu, Mei Hong, Shaoxian Chen, FTTH terminal connection technology and Products [J]. Telecommunications Technology (Phase 2): 62-65.

[13] Jian Liu, Quality Analysis of ODN Optical Passive Products. Telecommunications Science, 2013, (2): 129-134.
[14] Instruction Manual for Quality Sampling Inspection of the ODN Series Products of China Telecom Group in 2012, Released on July 2012.

[15] Xuehuang Lin, Optical Passive Device [M], Beijing, The People's Posts and Telecommunications Press (Posts \& Telecom Press). 